Research Article

Graeme Gillies*

\title{
Predictions of the shear modulus of cheese, a soft matter approach
}

https://doi.org/10.1515/arh-2019-0006

Received Sep 30, 2018; accepted Jun 27, 2019

\begin{abstract}
The rheological and structural properties of cheese govern many physical processes associated with cheese such as slumping, slicing and melting. To date there is no quantitative model that predicts shear modulus, viscosity or any other rheological property across the entire range of cheeses; only empirical fits that interpolate existing data. A lack of a comprehensive model is in part due to the many variables that can affect rheology such as salt, $\mathrm{pH}$, calcium levels, protein to moisture ratio, age and temperature. By modelling the casein matrix as a series core-shell nano particles assembled from calcium and protein these variables can be reduced onto a simpler twodimensional format consisting of attraction and equivalent hard sphere volume fraction. Approximating the interaction between core-shell nano particles with a Mie potential enables numerical predictions of shear moduli. More qualitatively, this two-dimensional picture can be applied quite broadly and captures the viscoelastic behaviour of soft and hard cheeses as well as their melting phenomena.

Keywords: Cheese; Core-Shell nano-particles; Shear modulus; Melting; Phase Separation; Phase Diagram; Gels; Glasses
\end{abstract}

PACS: 82.70Dd, 61.43-j

\section{Introduction}

Over the years there have been many reports of the rheological and fracture properties of cheese, but the vast amount of data remains unconsolidated since there are few tools that allow meaningful comparisons and/or prediction. There has been reasonable success with macro-

\footnotetext{
^Corresponding Author: Graeme Gillies: Fonterra Research and Development Centre, Dairy Farm Road, Fitzherbert, Palmerston North, 4472, New Zealand; Email: Graeme.Gillies@fonterra.com; Tel.: +64 6350 4649; Fax +64 6356 1476; https://orcid.org/0000-0002-1348-3406
}

əopen Access. ๑ 2019 G. Gillies et al., published by De Gruyter. (cc) BY License scale models treating cheese like a composite material where fat is a filler in a homogenous protein (casein) matrix [1, 2]. However, there are no quantitative models for how the protein matrix responds to the "cheese-maker's tool-kit" namely the protein-to-moisture ratio, $\mathrm{pH}$, ionic strength and calcium activity ${ }^{1}$. The effects of these variables has been studied $[3,4]$ but these variables are often coupled making it difficult to compare results across different articles. This difficulty is only exasperated with cheese maturity since the enzymatic breakdown of proteins depends on age, storage temperature and the specific microflora used in the cheese making [5]. To date there has been only one attempt at a universal conceptual picture of the casein matrix [6], which was based on the dual binding model $[7,8]$. This application of the dual binding model provides a good review of the many factors that affect properties of cheese, notably, $\mathrm{pH}$ and calcium solubility and maturation. The review is, however, limited to qualitative discussion. The review suggested the casein matrix is a hydrophobic colloid with the interaction energy between molecules = electrostatic repulsion and attractions stemming from hydrophobic interactions, +/- charge bridges and cross-links from colloidal calcium phosphate, CCP. Increasing the temperature reduces the gel firmness as hydrophobic attractions cause the casein molecules to contract reducing the overlap with neighbouring caseins. Lucey et al. [6], suggested that the protein matrix melts once the attractive interactions were equal or less than electrostatic repulsions. This statement was perhaps intended in a broader qualitative manner since the authors acknowledged and discussed two opposing points in the same article. First, the cheese doesn't melt but becomes progressively softer with temperature and eventually flows. Second, one must consider the intrinsic thermal energy of the caseins. The model stops short of any quantitative predictions, which makes the model difficult to validate or invalidate. Hinrichs and co-workers $[9,10]$, point out that molecular interactions alone cannot describe the melting and attribute melting to a sol gel transition.

1 For simplicity, the combination of $\mathrm{pH}$, salt and calcium levels will be abbreviated as ionic composition. 
Cheese rheology is further complicated with $\mathrm{G}^{\prime}$ and $\mathrm{G}^{\prime \prime}$ showing a power-law dependence with frequency and time-temperature-superposition [11], however shift factors differed on cooling than on heating. Nonetheless a polymer analogy is often hypothesised but never demonstrated with great rigor. The food rheologist is then left with questions on the appropriate constitutive model to predict behaviour in complex applications, for instance how to optimise cutting or grating, melting, or prevent cracking? Why is parmesan a brittle cheese while other cheeses are more ductile? Why doesn't moisture diffuse into the rinds where moisture is lower?

Various models consider the interior of the casein micelle as large disordered "aggregate" of protein coated calcium particles, where particles are bound by their hydrophobicity [12]. Holt and coworkers demonstrated precipitated calcium phosphate adsorbed $\beta$-casein phosphopeptides [13] which would resemble a core-shell arrangement. The disordered interior structure of the casein micelle contains open regions void of particles and denser zones [14] is reminiscent of a particle gel with a kinetically trapped structure. The interaction between CCP calcium and phosphoseryl groups is essentially ionic and thus much stronger than hydrophobic interactions.

In cheese, it is known that calcium solubilises on a scale of weeks and months [15], but negligible change would occur during a typical material science experiment. Thus, from a modelling perspective the system could be treated as a series of core-shell nanoparticle "assemblies" interacting via hydrophobic interactions. This analogy allows comparisons with other nano materials especially particulate gels or glasses which are generally described by their effective hard sphere volume, and the attractive potential.

The notion of the casein matrix acting as like a nanomaterial or colloid is not novel and has been attempted before. These models typically view casein as a gel, and melting as a gel-sol transition $[9,10]$ or flow defined by an activation energy [16]. The term sol-gel transition is often used to describe a transition in macromolecular solutions such as gelatin, or the precipitation of silica and metal oxide particles from solutions of low molecular weight ingredients [17]. In particulate colloidal systems sol-gel transitions can be observed by a change in volume fraction [18] or surface forces [19]. In these cases, there is a clear transition from freely diffusing (sol), and therefore viscous, to an elastic structure with little to no diffusion (gel/glass). In these systems the melting point is sharp and unequivocal, unlike cheese where the melting point is often defined by small amplitude oscillatory rheology as the temperature that the $G^{\prime \prime}$ surpasses $G^{\prime}$ which is a frequency dependent observation dependent on the sample history [11].

In this article, the notion of the casein matrix acting as like a nano-material is rationalised. Rather than treat the different caseins and CCPs as separate bodies calcium and protein treated as hard nano-particles with a grafted polymer layer in a semi-poor solvent. The behaviour of such a core-shell nano-particles is rather predictable, allowing estimates for shear modulus and a comparison with various cheeses. Another such prediction is that the observation of phase separation [20], apparent time temperature superposition [11], and the elasticity in melted cheese is more consistent with a gel or glass moving in and out of a kinetically trapped state. A kinetically trapped gel or glass challenges the notion of a second order melting transition or sol-gel transition. Accordingly, the casein matrix will be treated as a gel or glass and the properties rationalised with attraction strength and volume fraction. The rheology of concentrated attractive gels and glasses is still a topic of active research, nonetheless sufficiently mature for this article.

By assuming a core-shell nano-particle structure that swells and contracts depending on the environmental conditions and ionic composition allows mathematical analysis and comparisons with many other materials. The polymer shell or corona in a poor solvent captures the hydrophobic interactions while the ionic composition defines the size of the assembly by changing the extent of anchoring of the protein to the calcium core as well as thickness of the protein corona presumably via electrostatic repulsions.

Generalising protein as polymer is always a risky strategy, overlooking its true complexity. However, even notional appreciation of interaction energies will help validate or falsify otherwise difficult to test hypotheses. This generalisation also allows quantitative traction while minimising the number of adjustable parameters. The goal of this article is to demonstrate that this conceptual model captures the essential physics and not generate values for the parameters. Although equations have de-emphasized, they are not inescapable and are added to show the origin of each estimate. Those knowledgeable of cheese chemistry will appreciate that the chemistry cannot be overlooked, but it would greatly lengthen this article, and a complete review is perhaps better appreciated in a standalone piece. The fitted values presented in this article are intended to be a sensible reflection of the physics and not fits as the complexities and subtleties of the chemistry were overlooked to gain broader conceptual traction. 


\section{Casein matrix as condensed phase of core shell particles}

The rheology of cheese can be appreciated by first comparing with the behaviour of hard particles on $\epsilon$ versus $\varphi_{H S}$ diagram, Figure 1 [21]. Here, $\varphi_{H S}$ is the equivalent hard sphere volume fraction of the calcium protein assemblies and $\epsilon$ is the maximum attraction between these assemblies. Values for $\epsilon$ can be rationalised and are a function of temperature, while $\varphi_{H S}$ needs to be derived from the interaction potential and the number of assemblies. In this article the attraction between core-shell assemblies is discussed relative to $\mathrm{k}_{B} \mathrm{~T}$, as is the convention in physics, rather than the chemist's convention of $\mathrm{kJ} / \mathrm{mol}$ allowing general trends without need to be certain of molecular mass.

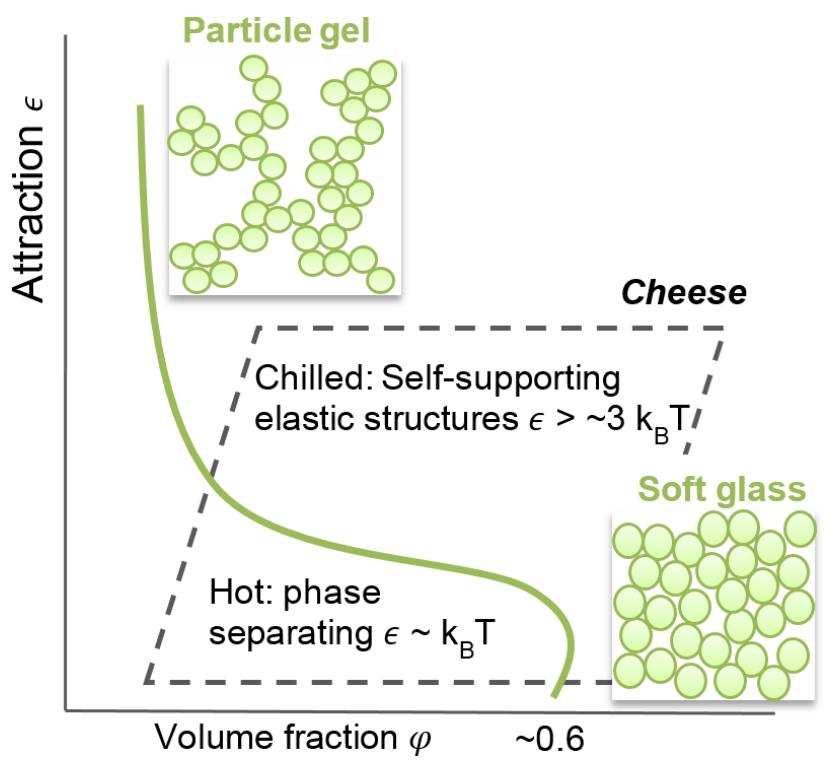

Figure 1: Phase diagram of hard particles, and the proposed positioning of cheese, adapted from Trappe and Sandkuhler [21].

Figure 1 shows that at low volume fractions, $\sim 10 \%$, and sufficiently strong attractions (typically $\epsilon>\sim 5 \mathrm{k}_{B} \mathrm{~T}$ ) an elastic network structure is formed. The strong attractions prevent particles from diffusing into local lower energy states. The same structure with weaker interactions sees occasional particles escaping the attraction of neighbours, but eventually particles become trapped in a lower energy state with more neighbours. The net result is the structure tends to evolve slowly, collapsing with domains rich and poor in particles; that grow with time. Decreasing $\epsilon$ further simply accelerates this phase separation process as particles are able to hop in and out of the energy wells at a greater rate. Pastorino et al. [20] demonstrated that heating a homogenous casein matrix heated to $50^{\circ} \mathrm{C}$ created micron-sized phase separated domains. The observation of larger but less numerous aggreagates, suggests the structure evolves sufficiently such that assemblies explore new energy wells, but ultimately find a new meta-stable state and evolution slows. This dynamic arrest suggests $\epsilon$ $=2 \mathrm{k}_{B} \mathrm{~T}-4 \mathrm{k}_{B} \mathrm{~T}$ depending on the volume fraction. Leaving soft and medium cheeses at $80^{\circ} \mathrm{C}$ for extended periods of time can result in substantial phase separation. This rapid structural rearrangement requires sufficiently low $\epsilon \sim 1$ $\mathrm{k}_{B} \mathrm{~T}$ otherwise assemblies are not able escape to the attractive pull of their neighbours. In this article $\epsilon \sim 3 \mathrm{k}_{B} \mathrm{~T}$ was chosen to describe cheese room temperature, values of 2 $\mathrm{k}_{B} \mathrm{~T}$ or $4 \mathrm{k}_{B} \mathrm{~T}$ would result in similar predictions.

\section{The size of a calcium-protein assembly}

\subsection{The calcium core}

The size of calcium protein assmebly can be broken down into two terms, the size of the CCP core; and the thickness of the casein corona. The exact composition of CCP appears to be as elusive as the structure of casein micelles. The reported molecular weight of CCP cores is approximately $10 \mathrm{kDa}$ [22] and described as brushite or hydroxyapatite. SAXS data suggests CCP cores have a radius around 2 $\mathrm{nm}$ [23]. This radius comes from casein micelle data rather than cheese data and the lower $\mathrm{pH}$ is expected to dissolve some of the calcium phosphate, so a smaller radius may be more appropriate. The composition of a range of available cheeses in local supermarkets reveals a calcium to protein mass-ratio of 1 to 35 with a standard deviation of 6. Osmotic stressing of skim milk suggests some form of sub structure within the casein micelle with a molecular weight of $156 \mathrm{kDa}$ and an equivalent hard sphere radius of 4.4nm [24]. $156 \mathrm{kDa}$ equates to $\approx 6$ caseins which will be assumed to be one core-shell calcium protein assembly. With the 1-35 mass ratio, six caseins per CCP particle equate to $18.5 \mathrm{kDa}$ brushite core, or a $10.8 \mathrm{kDa}$ core of hydroxyapatite, Figure 2 . The $10.8 \mathrm{kDa}$ results in core radii closer to that measured by SAXS, while the less dense brushite provides more surface area to accommodate the protein CCP stoichiometry. The surface area of the brushite or hydroxyapatite core does not accommodate adsorption of caseins assuming $\sim 3 \mathrm{mg} / \mathrm{m}^{2}$ regardless of molecular weight. Thus, the binding mechanism between a core and caseins is a grafting-type interaction via phosphoseryl residues in- 
teracting with the surface calcium groups. Such binding would depend on the ionic composition and the temperature [12, 25], much like the solubility of calcium phosphate. The $\alpha_{s 1}$ and $\alpha_{s 2}$ casein have multiple phosphoseryl residues and could bind at 1 or more places.

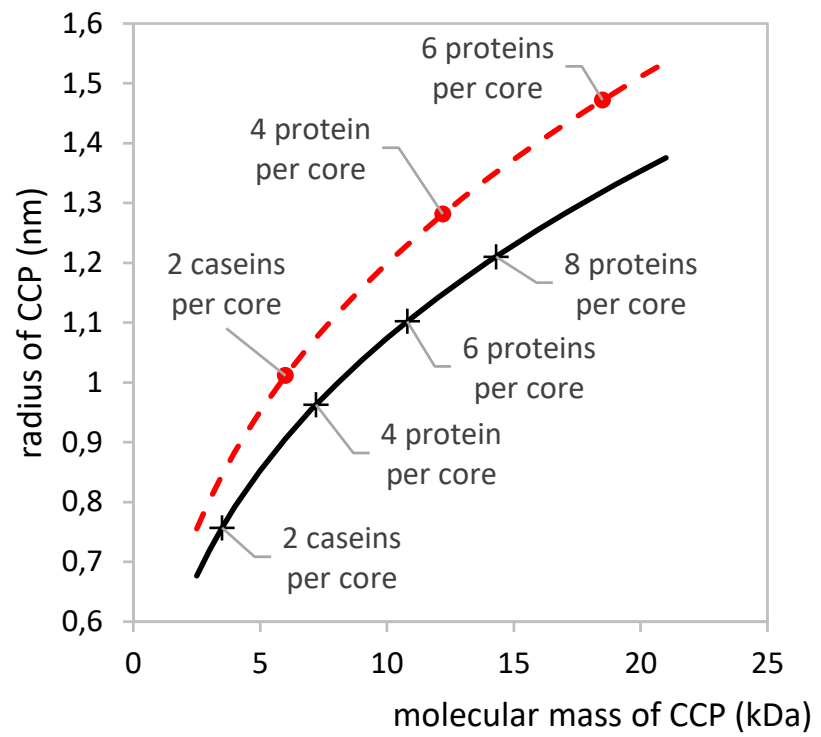

Figure 2: Radius of a CCP core calculated from its hypothetical molecular mass, marked points show stoichiometry assuming $1 \mathrm{~g}$ of calcium to $35 \mathrm{~g}$ of protein (molecular mass $25 \mathrm{kDa}$ ). Dashed red curve demonstrates a calculation based on a brushite core with $23 \%$ calcium by mass $\left(\mathrm{CaHPO}_{4} \cdot 2 \mathrm{H}_{2} \mathrm{O}\right)$ and density of $2300 \mathrm{~kg} / \mathrm{m}^{3}$. Solid black line demonstrates hydroxyapatite core $\mathrm{Ca}_{5}\left(\mathrm{PO}_{4}\right)_{3}(\mathrm{OH})$ with a density of $3200 \mathrm{~kg} / \mathrm{m}^{3}$.

These initial approximations overlook the kappa caseins fraction $(\sim 8 \%)$ which doesn't bind calcium, and assumes all calcium is in the CCP state. Accommodating a $72 \%$ insoluble casein observed in young cheddar [26], would drop the molecular weight of the core by $28 \%$, but the calculated radius would decrease by only $\approx 10 \%$. Naturally a binding state can be imagined where some tails detach completely from the core and it is certainly plausible that some caseins are only attached via weak interactions such as hydrogen bonding or hydrophobic interactions. Such weak binding is supported by the observation of $\beta$ - casein found in the serum of cheese extracted by centrifugation [27]. It becomes clear that multiple descriptions of a protein-calcium assembly are plausible. Rather than dwelling on many possibilities, the hypothesis is completed by fixing an $18.5 \mathrm{kDa}$ brushite core with a radius of $1.5 \mathrm{~nm}$ and six grafted caseins; half end grafted $\beta$ casein and half centre-grafted for $\alpha_{s 2}$ casein. This choice of parameters should not affect the generality of the hypothesis, but comparisons with other values are still valuable. It is also plausible that calcium-protein assemblies vary in size and mass, but accommodating this polydispersity has not been attempted.

\subsection{The outer protein corona}

The majority, $75 \%$, of the casein mass is $\alpha_{s 2}$ and $\beta$ casein which can be described with an approximate molecular mass of $25 \mathrm{kDa}$ consisting of 200 amino acids. Phosphoseryl groups are located in the hydrophilic centre of the $\alpha_{s 2}$ caseins and the hydrophilic end of $\beta$ casein. Binding of these phosphate groups to the calcium core orientates the hydrophobic tails outward. The centre-grafting of $\alpha_{s 2}$ casein creates two short tails while end grafting of $\beta$ casein results in a longer single tail. The interaction between calcium CCP sites and phosphoseryl groups is complex and depends on the ionic strength, calcium levels and pH. Conditions that favour complexation will essentially add mass and volume to the core while reducing the thickness of the corona. Complexation aside, electrostatic effects may also control the size of the corona [6]. Further adding to complexity is the effect of temperature. Temperature influences the hydrophobic interactions, with greater temperatures favouring a collapsed corona. The temperature's effect on the charge of the protein has not been considered.

The easiest to model cheese is the case where charges have little to no impact on the structure of protein-calcium assemblies with a minimum of grafting sites. When charge effects are negligible, the size of protein assemblies can be established using the approach of Daoud and Cotton [28] and has been applied to nano-particles with grafted layers [29]. Six caseins, $3 \alpha_{s 1}$ and $3 \beta$, are anchored onto a brushite core with radius $1.5 \mathrm{~nm}$, this gives a total of $\mathrm{f}=9$ arms, 6 from the $3 \alpha_{s 1}$ caseins and 3 from the $\beta$ caseins. For calculation simplicity, the 9 arms are further approximated as identical end grafted arms of $6 \times 200 / 9 \approx 130$ amino acids per arm. Amino acids are considered as identical non-descript rigid monomers and thus the number Kuhn segments per arm, $\mathrm{N}_{k}$ was taken as 130 . The Kuhn segment length, $1_{K}$ was assumed to be equal to the average amino acid diameter, $2 \mathrm{r}_{1}=0.64 \mathrm{~nm}$. The salt concentration in most cheeses is sufficiently high that the Debye length is reduced to the Bjerrum length, $\mathrm{l}_{B} \approx 0.7 \mathrm{~nm}$, which is fortuitously close to $l_{k}$ or $2 r_{1}$. Thus, individual charges only affect those on neighbouring monomers. However, interactions between two divalent phosphate groups will extend four times as far. The outer boundary radius $\mathrm{R}_{m}$ of assem- 
bly is given by Equation (1) [29]

$$
R_{m}=\left(\frac{8 N_{k} f^{\frac{(1-v)}{2 v}}}{3 v 4^{\frac{1}{v}}} L_{k}^{v}+R_{c c p}{ }^{v}\right)^{v}
$$

Where $v$ describes the solvent quality, and varies between 0.6 and 0.33 . This approach is not ideal for polar solvents such as water. Nonetheless, we can establish an interesting picture with $v$ between $v=0.5$ and $v=0.33$. For a poor solvent where $v=0.33$ polymer segments collapse in a densely packed structure and $\mathrm{R}_{m}$ is $3.5 \mathrm{~nm}$. A theta solvent $v=0.5, \mathrm{R}_{m}=7.4 \mathrm{~nm}$ describes ideal chain behaviour and represents the upper asymptote where hydrophobic forces can be present. Under conditions where calcium phosphate interactions promote binding to the core, smaller values of $\mathrm{N}_{k}$ would be expected, but $\mathrm{R}_{c c p}$ would need to grow to account for the anchored casein mass. The calculation presented here isn't particularly sensitive to the input parameters: repeating this calculation with a $10 \mathrm{kDa}$ hydroxyapatite core with 3 proteins giving 5 arms of 120 amino acids shows a smaller extreme gives a range of 2.7 and $6.2 \mathrm{~nm}$. These upper and lower limits of $\mathrm{R}_{m}$ can be used to establish the bounds of the interaction potential since the interaction should not extend beyond where assemblies are just touching, $2 \mathrm{R}_{m}$, while $\mathrm{R}_{H S}$ cannot drop below $\operatorname{R}_{m}(v=0.33)$ where the condensed assemblies would tend to hard sphere behaviour.

\section{The interactions between assemblies}

The interaction between colloidal particles with grafted layers are well understood but is not applicable in this instance. Interactions in colloid science assume the length scale the interaction is much smaller than the radius of the particles. In this instance the interaction length scale is twice the thickness of the corona which is comparable or larger than the calcium core radius. Consequently the interactions between assemblies can not be described by those typically used in colloid science [30, 31].

A Mie potential (Equation (2), Figure 3) is a convenient approach for handing short range repulsions and longer ranged attractions where the exact force law is not known. Unfortunately, the Mie potential has four adjustable parameters, $\mathrm{n}, \mathrm{m}, \sigma$ and $\epsilon$, that have to be determined or rationalised.

$$
U(d)=\frac{n}{n-m}\left(\frac{n}{m}\right)^{\frac{m}{n-m}} \epsilon\left(\left(\frac{\sigma}{d}\right)^{n}-\left(\frac{\sigma}{d}\right)^{m}\right)
$$

Here, $U(d)$ is the interaction potential as a function of the centre to centre separation $d . m$ and $n$ are unitless vari- ables that describe the curvature of the potential and $\sigma$ is the separation where the interaction potential equals zero, see Figure 3. $\mathrm{U}(\mathrm{d})$ and $\epsilon$ have units of energy.

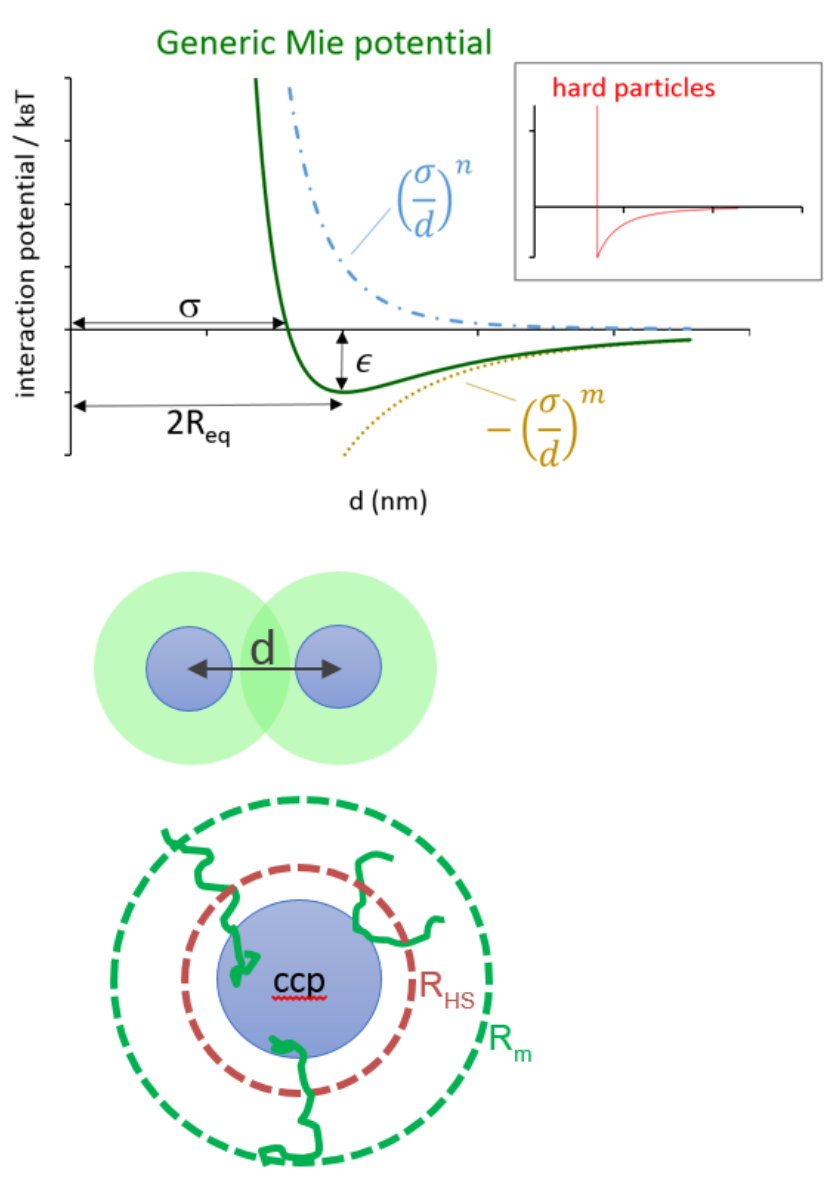

Figure 3: Schematic of the Mie potential and its repulsive and attractive components contrasted against an analogous hard particle system. The structure of the calcium protein assembly, and the symbols describing it. For visual clarity the protein - calcium assembly is drawn with only 3 proteins to give $f=4$ arms.

Star polymers may be a more appropriate analogy than colloids. Expressions for the interaction between star polymers with many arms in good and theta solvents are established [32]. The behaviour is defined by the number of polymer arms on each star, with stronger elastic repulsions observed with increasing number of arms. In doing so, particle cores with larger polymer shells are often considered the same manner as star polymers [29]. In effect the core is essentially a large monomer. Witten and Pincus [33] examined one and two-armed systems. It was suggested that a two arm system acts like a polymer with twice the number of monomers as a single arm. Likewise, des Cloizeaux [34] suggested the position of two monomers separated by a polymer chain also described the position 
between monomers on separate chains. In this article I propose the separation of two calcium cores can be approximated by the size of a polymer equal to twice the molecular weight of a casein arm. This hypothesis is rationalised further by the notion that overlapping hydrophobic tails will likely have many, albeit weak, connection points. The dimension of a single polymer chain was given by Flory [35]. Notably, under poor solvent conditions, two and three body interactions dominate entropic effects and the volume occupied by the polymer only contains small amounts of solvent. These two and three body interactions take on $\mathrm{a} \mathrm{d}^{-3}$ and $\mathrm{d}^{-6}$ relation and their sum resembles an $\mathrm{m}=3$ and $\mathrm{n}=6 \mathrm{Mie}$ potential. While the grounds for 3-6 Mie potential may be limited and difficult to validate, these values should capture the essential physics and provide calculable traction to progress the hypothesis. Notably, $\sigma$ and $\epsilon$ have the greatest significance and can be inferred from experiment. The value of $\mathrm{n}$ has greatest effect when calculating the modulus of compressed structures like cheddar and parmesan, while $\mathrm{m}$ defines structural rearrangement due to strain.

\subsection{Initial estimation of $\varphi_{H S}$ and $\epsilon$}

It was established earlier that $\mathrm{R}_{H S}$ must lie between the limits of $\mathrm{R}_{M}$ calculated with $v=0.33$ and 0.5 . For demonstrating the hypothesis, the average hard sphere of a room temperature cheese is assumed to be $5+/-0.5 \mathrm{~nm}$, noting that variations in protein hydration with ionic composition are well known [4]. It is worth noting the osmotic stressing experiments of Bouchoux et al. [24] indicate $\mathrm{R}_{H S}=4.4 \mathrm{~nm}$ in skim milk. The hard sphere radius can be calculated from Mie potential via the approach of Weeks et al. [36]. In this case, hard sphere radii were assumed allowing $\sigma$ to be fitted. Simple calculus reveals that the hard sphere radius and the equilibrium radius, $\mathrm{R}_{e q}$ are related and not sensitive to small changes in $\epsilon, \mathrm{m}$ or $\mathrm{n}$ values. Experiments of casein micelles [37] and acid casein [38] shows shrinkage with increasing temperature; in this model assemblies cannot shrink beyond a hard sphere radius of $v=0.33, \mathrm{R}_{H S}=$ 3.5nm.

The number of calcium-protein assemblies per unit mass of protein can be calculated from the molecular mass of protein per assembly. Likewise, the volume of the casein matrix per unit mass of protein can be approximated from the mass of each component and bulk densities of each component. A bulk density $\sim 1200 \mathrm{~kg} / \mathrm{m}^{3}$ was assumed for protein. From these two parameters a number concentration of assemblies, $\mathrm{N}$, can be calculated and then allows $\varphi_{H S}=\mathrm{N} 4 \pi \mathrm{R}_{H S}^{3} / 3$. It is quite instructive to create a graph of protein to moisture ratio versus $\varphi_{H S}$ and marking the position of soft, medium and hard cheeses, Figure 4a. It's known that protein to moisture ratio dominates the modulus, but it's now clear how different hard sphere radii arising from different ionic composition could outweigh small changes in the protein to moisture ratio. Notably, cheddar can be firmer than Emmantaller if its hard sphere radius is sufficiently larger despite a lower protein to moisture ratio. It's important to note that cheeses such as parmesan may have hard volume fraction well over the glass volume fraction suggesting calcium-protein particles inter-digitate beyond the hard sphere radius. This inter-digitation is perfectly acceptable within the model since the hard sphere radius represents the separation accessible to particles diffusing in and around the energy minimum. Closer separations would be achieved by applying work or by removing moisture when particles are in shrunken state and then allowing them to expand.

\subsection{Shear Modulus}

The response to strain in cheese varies strongly with the protein to moisture ratio and can be part rationalised with an understanding of $\varphi_{H S}$. The simplest models applied to gels don't consider any structural terms, for instance, $G^{\prime}$ $\sim \varphi_{H S} \mathrm{U}$ " $\left(2 \mathrm{R}_{e q}\right) / \mathrm{R}_{H S}$ [39] would give shear moduli between $120 \mathrm{kPa}\left(\mathrm{R}_{H S}=4.5 \mathrm{~nm}\right)$ and $220 \mathrm{kPa}\left(\mathrm{R}_{H S}=5.5 \mathrm{~nm}\right)$ at a protein to moisture ratio of 0.35 (unrippened camembert) which is a reasonable estimate [40, 41], but would tend to overestimate moduli for smaller values of $\varphi_{H S}$. In this equation, $\varphi_{H S}$ and $\mathrm{m}$ define the modulus of these gels. Weitz and co-workers [42, 43] have shown that colloidal gels far below the glassy limit scales as a power law function of the volume fraction $G^{\prime} \propto \varphi_{H S}{ }^{b}$ where $\mathrm{b}=3.2-3.9$. This power law scaling of volume fractions represents experimental data of cheese well for any value of $b$ between 3.2 and 3.9 with a prefactor of $10^{6} \mathrm{~Pa}$, Figure 4(b). Here it is important to compare trends in experimental data against the predicted curvature since $10^{6} \mathrm{~Pa}$ is only fit value. Unfortunately, there is very little shear modulus data on fat free cheeses to validate hypothesises presented here. The strategy taken to capture broader trends was to consider the far more popular compression modulus at small strains, Figure $4 \mathrm{~b}-4 \mathrm{c}$. The exact relation between initial compression modulus and shear modulus depends on the Poisson ratio, but in broad terms the compression modulus is approximately half of the shear modulus. Conversely, Yang et al. [1] found that cheeses have moduli two to three time greater than the matrix at $20^{\circ} \mathrm{C}$ when for typical fat levels of $20-30 \%$ (by mass). These two opposing effects allows a 

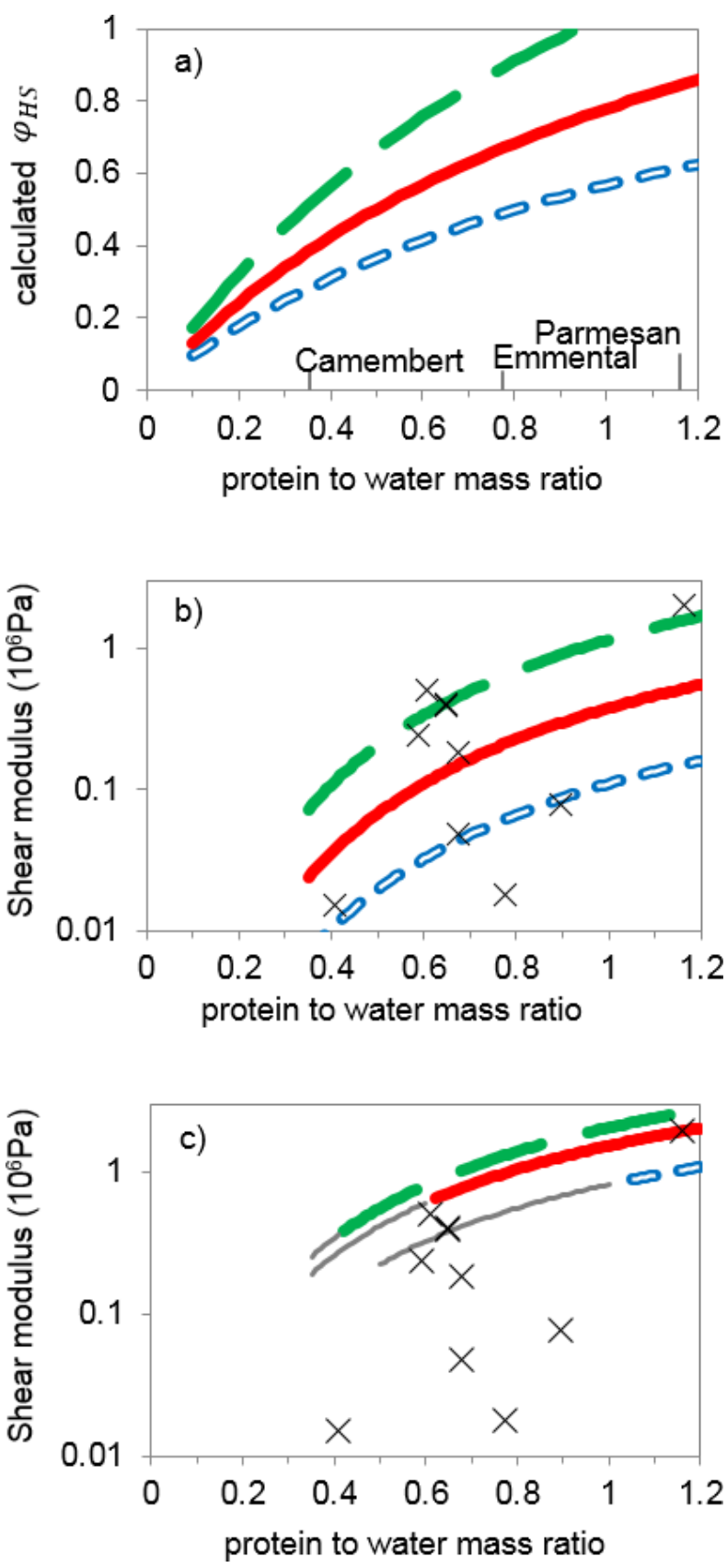

Figure 4: Calculations derived from protein to water mass ratio. 4a) Hard sphere volume fraction. 4b) Calculated shear modulus of the protein matrix calculated assuming $G^{\prime} \sim A \varphi_{H S}{ }^{b}$ where $\mathrm{A}=$ $10^{6} \mathrm{~Pa}$ and $b=3.5 .4 \mathrm{c}$ ) shear modulus of the protein matrix calculated from Evans and Lips [46]. Crosses indicate initial (low strain) compression modulus of cheeses obtained from reference [41] and references within; where data sample multiple ages were given, the data from the youngest sample was used. Lines indicate calculations using different hard sphere radii: dashed green line $5.5 \mathrm{~nm}$, solid red line $5.0 \mathrm{~nm}$, hollow blue rectangles $4.5 \mathrm{~nm}$. Figure $4 \mathrm{c}$ ) grey lines show the extrapolated calculation below the glass transition to demonstrate curvature but have no meaning. simple albeit approximate comparison between low strain compression data of cheeses and the predicted shear modulus of the protein matrix.

A more suitable model for the gel-like behaviour of the protein matrix would need to accommodate a structuredefining correlation length [42] or radial distribution function [44] requiring further data or justification. Models that apply some form of structural terms exist in the low and high protein extremes of cheeses. Freshly renneted milk and cottage cheese are likely to be described by the framework established by van Vliet and Walstra [45] where deformation and fracture strain are governed by the macrostructure of flexible strands and junction points. The volume fraction of these open gel structures is defined by the voluminosity of the casein micelle, $\sim 4 \mathrm{ml} / \mathrm{g}$, thus $\varphi \sim 14 \%$. The flexibility of connecting strands allows strain energy to dissipate without increasing the separation between neighbouring assemblies. Concentrating the protein further will progressively shorten strands reducing their flexibility and the length scale of the macro structure while creating a firmer structure $[42,45]$ and the value of $G^{\prime} / G^{\prime \prime}$ is expected to increase.

Increasing $\varphi_{H S}$ beyond $\sim 50 \%$ will see behaviour that is better described by colloidal glasses. At higher volume fractions still, the separations may be less than the hard sphere diameter. The interaction potential at these highvolume fractions is dominated by the repulsive component of the Mie potential. The shear modulus at these glasses can be taken from Evans and Lips [44] and is plotted on Figure 4c. Evans and Lips' were able to solve for Zwanzig and Mountain's equation [42] by restricting structural considerations to a particle immediate ( 11) neighbours. Evans and Lips analysis suggests the modulus is dependent primarily on the value of $\varphi_{H S}$ and $\mathrm{n}$ from the Mie potential via Equation (3). Small variations in the value of $\mathrm{n}$ result in substantial changes in the calculated modulus. Nonetheless the prediction envelope is consistent with the shear moduli of hard cheeses [41].

$$
G=N k_{B} T+\frac{\varphi_{c p} j}{5 \pi d^{\star}}\left(4 \frac{d U(d)}{d d^{\star}}+d^{\star} \frac{d^{2} U(d)}{d d^{\star 2}}\right)
$$

Here $j \sim 11$, is the number of neighbours surrounding each assembly, $\varphi_{c p}$ is the close packing volume fraction and was assumed to be $\sim 0.6$. Note that $\mathrm{d}^{\star}$ is the centre to centre separation, like $d$, the use of the * symbol is used to denote that the condition $\varphi \geq \varphi_{c p}$ and consequently $\mathrm{d}^{\star}$ is given by equation (4)

$$
d^{\star}=2 R_{e q}\left(\frac{\varphi_{c p}}{\varphi}\right)^{\frac{1}{3}}
$$

Zwanzig and Mountain's equation [44] assumes proteincalcium assemblies are not rearranging their positions. 


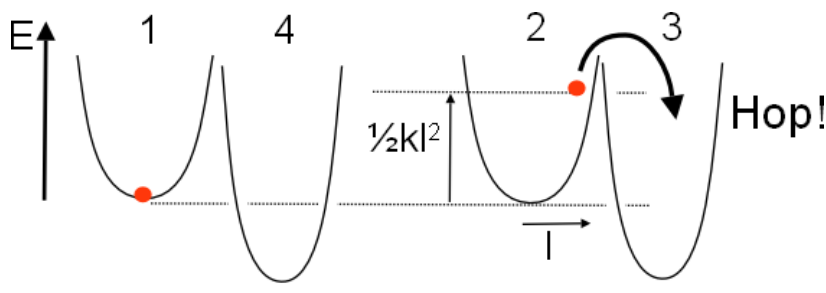

1

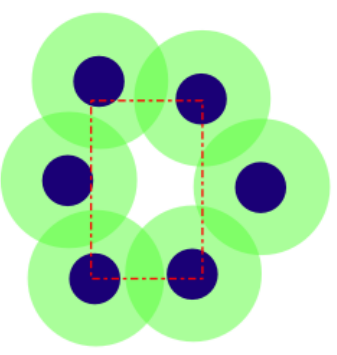

2
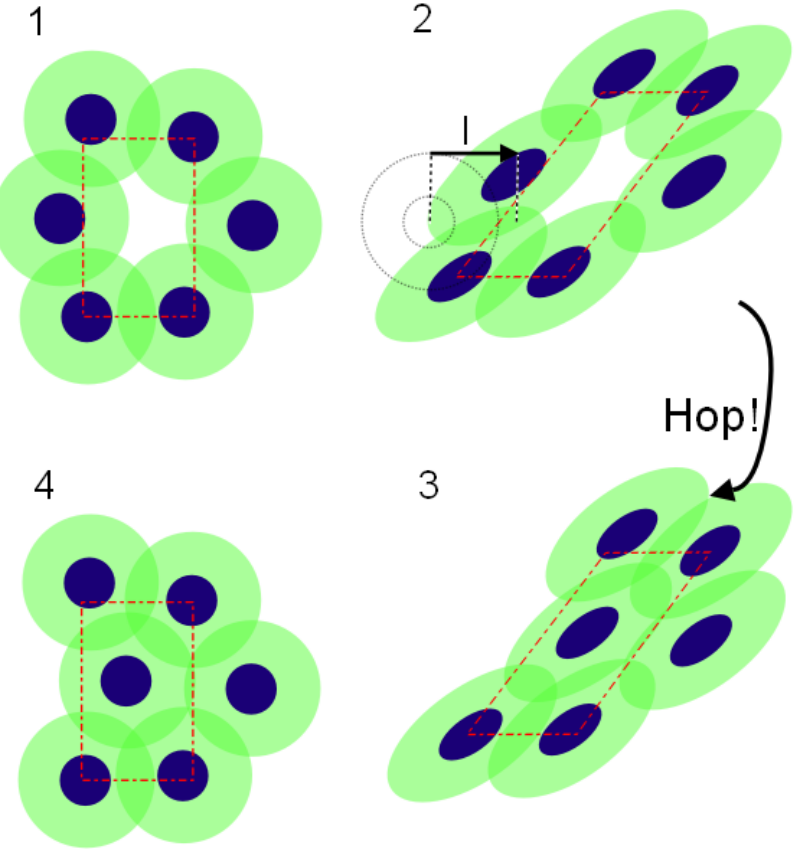

Figure 5: Schematic showing the hop and trap model for the arrangement of calcium - protein assemblies showing and analogous energy wells, adapted from Fielding et al. [49]. The schematic is intedended to be read clockwise, 1) local arrangement is meta stable, but diffusion from 1 to 4 is impossible. 2) Apply a strain decreases the remaining barrier height required for rearrangement. Diffusion into state 3 is possible with sufficient time. 3) Protein-calcium assemblies rearrange into a new energy well. 4, The applied strain is removed but rearrangements imply not all strain is recovered.

Slower frequencies must accommodate localised shear induced rearrangements, colloquially named "hops". "Hop and trap" models under no strain were first proposed by Bouchaud [47] and later extended by Sollich et al. [48]. These complex models suggest diffusion within a sample allows particles to explore different configurations but eventually get trapped in deeper energy states. The theory of soft glassy rheology is quite complex and the reader is pointed to a review on the subject [49]. In short, the energy required for such structural rearrangements generally exceeds the thermal energy, and particles are kinetically "traped". Diffusion out of a trap of $>20 \mathrm{k}_{B} \mathrm{~T}$ is not a meaningful concept and will be infinitely slow and superseeded by enzymatic breakdown of the caseins. A hop may occur if given sufficient strain energy, Figure 5. The

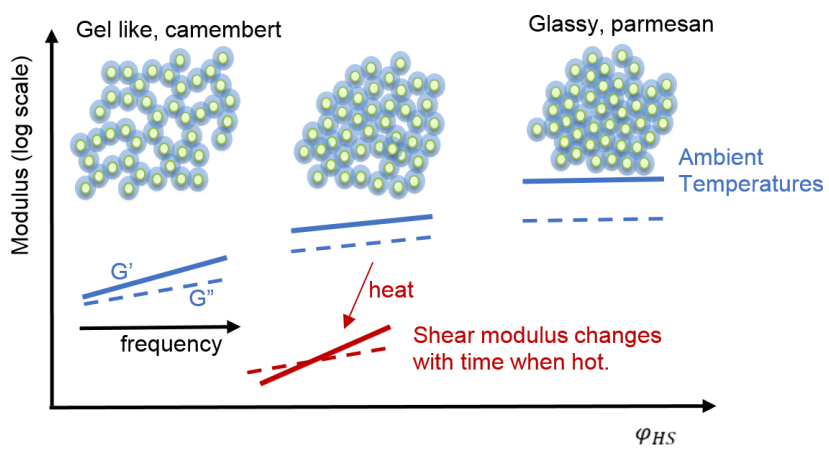

Figure 6: Schematic representation of the various structure of cheese as a function of volume fraction and the temperature and frequency dependence on the storage $\mathrm{G}^{\prime}$ and loss $\mathrm{G}^{\prime \prime}$ shear moduli. Solid blue and red lines represent shear storage modulus as a function of frequency at ambient and elevated temperature while dashed lines represent the shear loss modulus.

local strain energy is defined as $\mathrm{E} \approx \mathrm{kl}^{2}$ where $\mathrm{k}$ is a spring constant, analogous to a local modulus, and 1 is the local strain sufficiently small that the force distance relationship remains linear. Inutitively, local yielding occurs when the local strain energy exceeds that of the trap energy, but this need not be the case. The strain energy only needs to reduce the barrier height such that the particle is no longer kinetically trapped and the rate of hopping takes on an Arrhenius-like form. The rate at which hops occur depend on the number of potential hopping sites or analogously the volume fraction and the remaining activation barrier height. This hopping behaviour is consistent with compression-relaxation data from Masi and Addeo [50] who proposed that intermolecular links would break reform over time when under strain. A hop is the adsorption of energy, where the local strain and strain energy is reset to zero, or thereabouts. This adsorption manifest as a growth in $\mathrm{G}^{\prime \prime}$ at the expense of $\mathrm{G}^{\prime}$.

Another feature predicted by soft glassy rheology is $\mathrm{G}^{\prime}$ and $G^{\prime \prime}$ have a power law dependence on the frequency that is easy to rationalise in cheese. For a medium to hard cheese $\left(\varphi_{H S}>0.6\right)$ at low temperatures where the $\varphi_{H S}$ and $\epsilon$ are their greatest, the probability of a hop is equally small regardless of time and $\mathrm{G}^{\prime}$ is far greater than $\mathrm{G}^{\prime \prime}$. Reducing the voluminosity of the protein matrix via reducing the total protein content should increase the number of shear transformation sites and thus hops are more probable, especially at low frequencies and/or higher strains. Increasing the temperature decreases both $\varphi_{H S}$ and $\epsilon$. The decrease in $\varphi_{H S}$ decreases the modulus, while the decrease in $\varphi_{H S}$ and $\epsilon$ both increase the probability of hops. Thus $\mathrm{G}^{\prime}$ and $\mathrm{G}^{\prime \prime}$ show a steeper frequency dependence and the strain at which $\mathrm{G}^{\prime}$ drops decreases. At sufficiently high temperatures the energy barrier for hopping should be accesi- 
ble by diffusion alone allowing the sample to phase separate if $\varphi_{H S}$ is sufficiently low.

\section{Conclusion}

By treating cheese as an attractive glass of soft particles, the rheology of cheese can be predicted and rationalised in detail. By treating CCP particles and proteins as a collective body greatly reduces the need to follow many different interactions. Instead the key term needed to understand cheese behaviour is $\mathrm{R}_{H S}$ and how it may be changed.

A 3-6 Mie potential was used to generate shear modulus values that approximate cheese to the right order of magnitude for hard cheese. The moduli for soft cheeses are consistent, but structural information such as a pair correlation function is needed to be conclusive. Converting inter-particle interactions and/or $\varphi_{H S}$ to a shear modulus isn't particularly novel, and there exist many models to do so. Most significantly, an attractive core-shell nanoparticle model here provides a single mechanism understanding of different properties of the casein matrix. It is also a model that can be validated experimentally. X-ray Scattering experiments can be used to validate my initial estimates of $\mathrm{R}_{H S}$, but also provide structure information beyond $\varphi_{H S}$. Understanding cheese as a function of volume fraction and attraction depth unifies ideas such as protein hydration [4, 27], phase separation [20] and melting [11] and can explain an array of rheology data:

The ductile to brittle nature of cheeses can in part be explained by the effect of the hard sphere volume fraction of assemblies within the protein matrix. Increasing the $\varphi_{H S}$ progressively reduces the number of sites where shear induced rearrangements may occur and therefore the probability that local strains can be dissipated. One can speculate that this transition occurs near or around the glass transition. The glass transition is best described by a volume fraction but in its absence $G^{\prime}>10^{6}$ Pa serves as an approximate indicator of glassy behaviour.

Cutting and grating is often correlated with $\mathrm{G}^{\prime}$ and $\mathrm{G}^{\prime \prime}$ values [51]. While the cracking of holes in Swiss cheese is related to fracture strain [52]. In both cases, the ionic composition is known to be a controlling factor, but a single variable of $\varphi_{H S}$ reveals how the viscous dissipation can be controlled to allow or prevent crack propagation. Furthermore, in cutting and grating any flow would also result in greater contact areas and therefore friction. A related phenomenon is rubber-like friction and is reviewed elsewhere [53].
The formation of rinds from brining can be likened to a form of strain hardening. A rind forms on the cheese because the osmotic pressure draws water from the cheese surface into the brine bath [4] creating a local compression. After sufficient number shear transformations occur diffusion of water into the now firmer rind surface is now energetically unfavourable.

Melting, of the casein matrix, must come from two effects, the first is the shrinking effect of the caseins which reduces the modulus and the second from the rearrangement of caseins which will be slower, but would tend to increase the modulus. Fat globules reside in, and give volume to the aqueous domains which would assist fluid flow. The frequency dependence $\mathrm{w}^{p}$ of concentrated milk gels and cheese varies from about $\mathrm{p} 0-0.2[7,11,54]$ and increases up to $p \approx 1$ at $40-90^{\circ} \mathrm{C}[9,11,54]$ depending on composition. Thus, the frequency exponent isn't expected to change significantly as the structure evolves with time even though structural rearrangements will change the modulus noticeably. This slow variation in $\mathrm{n}$ casts doubt on any results claiming time - temperature superposition, as the shift factor will not have meaning, and will vary with sample history, as has already been noticed between heating and cooling [9]. Heating cheese will cause the formation of dense domains that are local glassy states which when cooled adsorb water much slower than they released water, creating structural difference before and after heating. This temperature dependence also suggests there is no melting point only a gradual change in flow characteristics that change with temperature and time and is not a sol-gel transition.

Softening due to enzymatic breakdown of a cheese is quite different to melting. Enzymatic breakdown isn't expected to change the hard sphere volume fraction significantly as the $\mathrm{R}_{m}$ is only a weak function of the size and number of arms. Nonetheless, enzymatic breakdown may break junctions between neighbouring particles allowing for more shear transformations thus decreasing $\mathrm{G}^{\prime}$ in favour of $\mathrm{G}^{\prime \prime}$. Rate constants for enzymatic breakdown are known and could be used to generate values for $\epsilon$ and/or $\mathrm{G}^{\prime}$ as a function of time.

Acknowledgement: This research was supported through the Dairy Primary Growth Partnership programme in Food Structure Design by Fonterra Co-operative Group Ltd. and the New Zealand Ministry for Primary Industries.

Skelte Anema provided significant insight and references that assisted in the writing this article. Kees (C.G.) De Kruif, Christina Coker, Steve Taylor, Philip Watkinson, David Reid, Peter Munro, Erich Windhab and E. Allan Foegeding are also acknowledged for their useful comments. 
Conflict of Interests: At the time of submission, the author had an patent application relating to pasta filata cheese WO2017111621A1

\section{References}

[1] Yang X., Rogers N.R., Berry T.K., Foegeding E.A., Modeling the rheological properties of cheddar cheese with different fat contents J. Texture Stud., 42, (2011) 331.

[2] Thionnet O., Havea P., Gillies G., Lad M., Golding M., Influence of the Volume Fraction, Size and Surface Coating of Hard Spheres on the Microstructure and Rheological Properties of Model Mozzarella Cheese, Food Biophysics, 12, (2017) 33.

[3] Brickley C.A., Lucey J.A., Mcsweeney P.H., Effect of the addi- tion of trisodium citrate and calcium chloride during salting on the rheological and textural properties of Cheddar-style cheese during ripening, Int. J. of Dairy Technol. 62, (2009) 527.

[4] Guinee T.P., Salting and the role of salt in cheese, Int. J. of Dairy Technol. 57, (2004) 99.

[5] Coker C.J., Crawford R.A., Johnston K.A., Singh H., Creamer L.K., Towards the classification of cheese variety and maturity on the basis of statistical analysis of proteolysis data-a review, Int. Dairy J. 15, (2005) 631.

[6] Lucey J.A., Johnson M.E., Horne D.S., Invited Review: Perspectives on the Basis of the Rheology and Texture Properties of Cheese, J. Dairy Sci., 86, (2003) 2725.

[7] Horne D.S., Casein Interactions: Casting Light on the Black Boxes, the structure in Dairy Products, Int. Dairy J., 8, (1998) 171.

[8] Horne D.S., Casein micelle structure: Models and muddles, Curr. Opin in Colloid and Interface Sci., 11, (2006) 148.

[9] Bähler B., Back R., Evaluation of oscillatory and shear strain behaviour for thermorheological plasticisation of non-ripened cheese curd: Effect of water, protein, and fat J. Hinrichs, Int. Dairy J. 46, (2015) 63.

[10] Schenkel P., Samudrala R., Hinrichs J., Thermo-physical properties of semi-hard cheese made with different fat fractions: Influence of melting point and fat globule size, Int. Dairy J., 30, (2013) 79.

[11] Udyarajan C.T., Horne D.S., Lucey J.A., Use of time-temperature superposition to study the rheological properties of cheese during heating and cooling, Int. J. Food Sci. and Technol., 42, (2007) 686.

[12] Dalgleish D.G., Parker T.G., Binding of calcium ions to bovine alpha s1-casein and precipitability of the protein-calcium ion complexes. J. Dairy Res. 47, (1980) 113.

[13] Holt C., Timmins P.A., Errington N., Leaver J., A core-shell model of calcium phosphate nanoclusters stabilized by beta-casein phosphopeptides, derived from sedimentation equilibrium and small-angle X-ray and neutron-scattering mea- surements, Eur. J. of Biochem., 252, (1998) 73.

[14] Bouchoux A., Geśan-Guiziou G., Peŕez J., Cabane B., How to Squeeze a Sponge: Casein Micelles under Osmotic Stress, a SAXS Study, B., Biophys. J. 99, (2010) 3754.

[15] Hassan A., Johnson M.E., Lucey J.A., Changes in the Proportions of Soluble and Insoluble Calcium During the Ripening of Cheddar Cheese, J. Dairy Sci., (2004) 854.
[16] Tunick M.H., Activation energy measurements in rheological analysis of cheese, Int. Dairy J., Volume 20, (2010) 680.

[17] Gauthier-Manuel B., Guyon E., Roux S., Gits S., Lefaucheux F., Critical viscoelastic study of the gelation of silica particles, J. de Phys., 48, (1987) 869.

[18] Scheffold F., Diáz-Leyva P., Reufer M., BenBraham N., Lynch I., Harden J.L, Brushlike Interactions between Thermoresponsive Microgel Particles, Phys. Rev. Lett., 104, (2010) 128304.

[19] Romer S., Scheffold F., Schurtenberger P., Sol-Gel Transition of Concentrated Colloidal Suspensions, Phys. Rev. Lett., 85, (2000) 4980.

[20] Pastorino A.J., Dave R.I., Oberg C.J., McMahon D.J., Temperature Effect on Structure-Opacity Relationships of Nonfat Mozzarella Cheese, J. Dairy Sci., 58, (2002) 2106.

[21] Trappe V., Sandkuhler P., Colloidal gels-low-density disordered solid-like states, Curr. Opin. Colloid and Interface Sci., 8, (2004) 494.

[22] Choi J., Horne D.S., Lucey J.A., Determination of molecular weight of a purified fraction of colloidal calcium phosphate derived from the casein micelles of bovine milk, J. Dairy Sci. 94, (2011) 3250.

[23] deKruif C.G., Huppertz T., Urban V.S., Petukhov A.V., Advances in Colloid and Interface Sci. 171-172, (2012) 36.

[24] Bouchoux A., Cayemitte P-E., Jardin J., Gesan-Guiziou G., Cabane B., Casein Micelle Dispersions under Osmotic Stress, Bio- phys. J., 96, (2009) 693.

Erratum. Bouchoux A., Cayemitte P-E., Jardin J., Gesan-Guiziou G., Cabane B, B., Biophys. J. 96, (2009) 2547.

[25] Parker T.G., Dalgleish D.G., Binding of calcium ion stobovine beta-casein., J. Dairy Res. 48, (1981) 71.

[26] Lucey J.A., Mishra R., Hassan A., Johnson M.E., Rheological and calcium equilibrium changes during the ripening of Cheddar cheese, Int. Dairy J., 15, (2005) 645.

[27] Guo M.R., Kindstedt P.S., Age-Related Changes in the Water Phase of Mozzarella Cheese, J. Dairy Sci., 78, (1995) 2099.

[28] Daoud M., Cotton J.P., Star shaped polymers: a model for the conformation and its concentration dependence, J. de phys. Paris, 43, (1982) 531.

[29] Mefford O.T., Vadala M.L., Goff J.D., Carroll M.R. J., Mejia-Ariza R., Caba B.L., St. Pierre T.G., Woodward R.C., Davis R.M., Riffle J.S., Stability of Polydimethylsiloxane-Magnetite Nanoparticle Dispersions Against Flocculation: Interparticle Interactions of Polydisperse Materials, Langmuir, 24, (2008) 5060.

[30] de Gennes P-G., Polymers at an interface. 2. Interaction between two plates carrying adsorbed polymer layers, Macromolecules, 15 (1982) 492.

[31] Vincent B., Edwards J., Emmett S., Jones A., Depletion Flocculation in Dispersions of Sterically Stabilised Particles ("Soft Spheres"), Colloids and Surfaces, 18, (1986) 261.

[32] Likos C.N., Effective Interactions in Soft Condensed Matter Physics, Phys. Reports, 348 (2001) 267.

[33] Witten T.A., Pincus P.A., Colloid Stabilization by Long Grafted Polymers Macromolecules, 19, (1986) 2509.

[34] des Cloizeaux J., Short range correlation between elements of a long polymer in a good solvent, J. de Phys., 41, (1980) 223.

[35] Flory P.J., Thermodynamics of High Polymer Solutions, J. Chem. Phys. 10, (1942) 51.

[36] Weeks J.D., Chandler D., Andersen H.C., Role of Repulsive Forces in Determining the Equilibrium Structure of Simple Liquids, J. Chem. Phys. 54, (1971) 5237. 
[37] Nob̈el S., Weidendorfer K., Hinrichs J., Apparent voluminosity of casein micelles determined by rheometry, J. Colloid and Interface Sci, 386, (2012) 174.

[38] O'Meara G.M., Munro P.A., The Precipitation and Shrinkage of Acid Casein Curd: A Preliminary Study. New Zealand J. of Dairy Sci. Technol., 17, (1982) 147.

[39] Larson R.G., The structure and rheology of complex fluids. (New York: Oxford University Press 1999).

[40] Pagana M.M., Hardy J., Effect of Salting on Some Rheological Properties of fresh Camembert Cheese as Measured by Uniaxial Compression, Milchwissenschaft, 41, (1986) 210.

[41] Gunasekaran S., Mehmet Ak M., Cheese Rheology and Texture (CRC press 2002).

[42] Krall A.H., Weitz D.A., Internal Dynamics and Elasticity of Fractal Colloidal Gels, Phys. Rev. Lett., 80, (1998) 778.

[43] Gisler T., Ball R.C., Weitz D.A., Strain Hardening of Fractal Colloidal Gels, Physical Review Letters, 82, (1999) 1064.

[44] Zwanzig R., Mountain R. D., High Frequency Elastic Moduli of Simple Fluids, J. Chem. Phys., 43, (1965) 4464.

[45] van Vliet T., Walstra P., Large deformation and fracture behaviour of gels, Faraday Discussions, 101, (1995) 359.
[46] Evans I.D., Lips A., Concentration dependence of the linear elastic behaviour of model microgel dispersions, J. of the R. Chem. Soc, Faraday Trans., 86, (1990) 3413.

[47] Bouchaud J.P., Weak ergodicity breaking and aging in disordered systems, J. de Phys. I, 2 (1992) 1705.

[48] Sollich P., Lequeux F., Heb́raud P., Cates M.E., Physical Review Letters, Rheology of Soft Glassy Materials, 78, (1997) 2020.

[49] Fielding S.M., Sollich P., Cates M.E., Aging and rheology in soft materials, J. Rheology, 44, (2000) 323.

[50] Masi P., Addeo F., An examination of some mechanical properties of a group of Italian cheeses and their relation to structure and conditions of manufacture, J. Food Eng. 5, (1986) 217.

[51] Childs J.L., Daubert C.R., Stefanski L., Foegeding E.A., Factors Regulating Cheese Shreddability, J. Dairy Sci. 90, (2007) 2163.

[52] Law B.A., Tamime A.Y., Technology of Cheesemaking, (Shefleld: Shefleld Academic Press, 1999).

[53] Persson B.N.J., On the theory of rubber friction, Surface Science, 401, (1998) 445.

[54] Muliwan E.B., Hatzikiriako S.G., Rheology of Mozzarella cheese, Int. Dairy J., (2007) 1063. 